\title{
The Healing Effect of Platelet-rich Plasma (PRP) Jelly in Rabbits Undergoing Tracheal Resection and Anastomosis
}

\author{
JAE YEON LEE ${ }^{1}$, SOO JIN SON ${ }^{2}$, SEOK HWA CHOI ${ }^{3}$ and DONG-WOO CHO ${ }^{1}$ \\ ${ }^{1}$ Department of Mechanical Engineering, POSTECH, Pohang, Republic of Korea; \\ ${ }^{2}$ Korea Mouse Phenotyping Center, Seoul, Republic of Korea; \\ ${ }^{3}$ Veterinary Medical Center, Chungbuk National University, Cheongju, Republic of Korea
}

\begin{abstract}
Background/Aim: Tracheal anastomosis is commonly indicated for patients with tracheal tumor, stenosis and tracheoesophageal fistula. This study was designed to evaluate the healing activity of topical application of platelet-rich plasma $(P R P)$ jelly for tracheal reconstruction in a rabbit model. Materials and Methods: Thirty-six healthy New Zealand White rabbits were assigned to three groups of 12 animals each. The control group underwent tracheal resection and anastomosis. The fibrin-glue group and PRP-jelly group underwent tracheal resection and anastomosis plus fibrin glue or PRP jelly application over the anastomosis and surgical field, respectively. Results: The PRP jelly treatment enhanced the healing capacity by positive effects on healing rate, tissue fill, and volume fraction of fibroblasts during the 28 days following surgery. Conclusion: Application of PRP jelly to tracheal anastomotic wounds can accelerate or improve the quality of repair.
\end{abstract}

Tracheal resection and reconstruction is commonly performed for patients with tracheal obstruction in order to correct various forms of tracheal stenosis. Common causes of tracheal obstruction include foreign bodies and tracheal tumors. Tracheal resection with end-to-end reconstruction is an alternative treatment (1-4). However, its postoperative complications range from minimal granulation and stenosis to the most fatal complications, including total disruption of the anastomosis due to ischemia (5).

The healing process after airway anastomosis may affect patient outcome. Several topical applications of growth factors can improve wound healing. An autologous platelet

This article is freely accessible online.

Correspondence to: Dong-Woo Cho, Department of Mechanical Engineering, POSTECH, Pohang 790-784, Republic of Korea. Tel: +82 542792171, Fax: +82 542795419, e-mail: dwcho@ postech.ac.kr

Key Words: Healing, platelet-rich plasma jelly, rabbit, tracheal anastomosis. concentrate suspended in plasma (platelet-rich plasma, PRP) is a treatment option that promotes wound healing (6). A recent study found that autologous platelet concentrate with growth factors or PRP may accelerate wound healing of tracheal anastomosis in a pig model (7).

This study evaluated the wound healing effect of PRP jelly compared to fibrin glue, a formulation widely used to create a fibrin clot at the wound site, for tracheal reconstruction in a rabbit model.

\section{Materials and Methods}

Thirty-six healthy New Zealand White rabbits $(3.2 \pm 0.6 \mathrm{~kg}, 4$ to 5 months old) were used in this study. The protocol for the animal experiment was approved by the Laboratory Animal Research Center of Chungbuk National University (Approval No. 714-14-01). All rabbits were housed in individual cages throughout the experimental period. Water and food were supplied ad libitum. Animals were divided into three groups with 12 animals each: Group 1 underwent surgery only, group 2 underwent surgery and fibrin glue application to the anastomosis, and group 3 underwent surgery and application of PRP jelly to the anastomosis. All animals were observed for potential complications related to tracheal resection and anastomosis. Half of the animals were sacrificed on day 14 and the other half on day 28 following euthanasia guidelines for experimental animals. These guidelines are adapted from the report of the American Veterinary Medical Association Panel on Euthanasia (8).

Five milliliters of blood were obtained from each rabbit, mixed gently with $0.5 \mathrm{mI}$ of the anticoagulant citrate dextrose A (Cytosol Laboratories, Braintree Co., Braintree, MS, USA) and transferred to a blood chamber. Another $1 \mathrm{mI}$ of citrate dextrose A was injected into the plasma chamber. The anticoagulated blood was centrifuged to separate the platelet-containing plasma from the red blood cells. To produce PRP jelly, the platelets were activated by addition of 5 $\mathrm{ml}$ of $10 \%$ calcium chloride (Calcium Chloride Injection, USP 10\%; American Regent, Shirley Co., NY, USA) and 5,000 U bovine thrombin powder (Jones Pharm, Bristol Co., St Louis, MO, USA).

Rabbits were anesthetized with a cocktail of $35 \mathrm{mg} / \mathrm{kg}$ ketamine (ketamine50 Inj., Yuhan Co., Seoul, Korea) and $5 \mathrm{mg} / \mathrm{kg}$ xylazine (Rompun, Bayer Korea Co., Seoul, Korea) administered intramuscularly. The skin was shaved and cleaned with a mixture of iodine and $70 \%$ ethanol. A midline incision of skin and subcutaneous tissue was made from $1 \mathrm{~cm}$ below the larynx to a length of $6 \mathrm{~cm}$. The entire 
cervical trachea was exposed after ventral cervical incision. Two centimeters of the trachea were resected, and the trachea was end-toend anastomosed using interrupted simple $5 / 0$ polydioxanone sutures. In group 2 fibrin glue (TISSEEL ${ }^{\text {тм }}$ Kit; Baxter India, Haryana, India) and in group 3, PRP jelly was applied over the anastomosis and surgical field. All animals were administered antibiotic intramuscularly (20 mg/kg amoxicillin, Foxolin Inj.; Samjin Pharm. Co., Seoul, Korea) and analgesic ( $3 \mathrm{mg} / \mathrm{kg}$ tramadol, Tamadol Inj.; Dongkwang Pharm. Co., Seoul, Korea) for 7 days.

The rabbits were observed daily for evaluation of respiratory distress including coughing and signs of dyspnea. Radiographs of animals were taken immediately prior to sacrifice at either 14 or 28 days, and the percentage of luminal stenosis was measured in all animals at postmortem examination. The surgical sites were evaluated for adhesion using the following scoring system: Score 0 , no adhesion; score 1, thin filmy adhesion; score 2, more than one thin adhesion; score 3 , thick adhesion with focal point; score 4 , thick adhesion with planar attachment; score 5, very thick vascularized adhesions or more than one planar adhesion (9).

Tracheas were embedded in paraffin, and slides were stained with hematoxylin and eosin. Tracheal structure, vascular density, and inflammatory cell infiltration were evaluated by a pathologist blinded to the treatment modalities. Tracheal revascularization was quantified by the number of vascular structures included in three randomly selected fields per slide, viewed at 10x magnification.

All data were recorded as means and standard deviation. Kruskal-Wallis analysis of variance was performed for group comparisons. Significance of differences among the groups was determined by a nonparametric Mann-Whitney $U$-test. Differences within groups were tested with one-way analysis of variance (ANOVA) and Duncan's post-hoc tests if ANOVA gave significant results. Values of $p<0.05$ were considered statistically significant. All statistics were analyzed with SPSS software ver. 22.0 (IBM Corp., Armonk, NY, USA).

\section{Results}

All animals survived to the end-point except one rabbit in group 1 that died of asphyxia from tracheal rupture at 13 days post-surgery. The inner diameter of the trachea at the anastomotic site (percentage of luminal stenosis) was measured and compared to the diameter of normal trachea in the same specimen. No local or systemic complications due to PRP jelly or fibrin glue application were detected, and post-mortem examinations revealed no evidence of anastomotic complications. Some animals coughed intermittently, and one animal in group 1 showed breathing abnormality prior to death. Some breathing noises were observed in two animals in group 1, which had $44 \%$ and $43.8 \%$ stenosis (Table I). Adhesion scores were significantly lower in the PRP jelly and fibrin glue-treated groups compared with the control group, but there was no significant difference between the PRP jelly-and fibrin gluetreated groups (Figure 1). Adhesion scores were lower in the treatment groups than the control group. In addition, the adhesion formation rate was significantly lower in the PRP jelly-treated group compared with the other groups. These

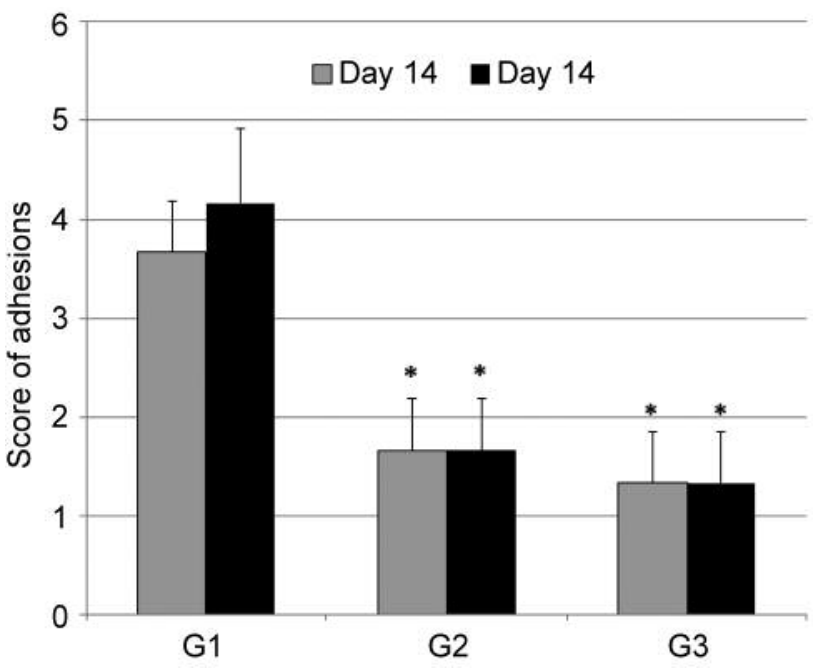

Figure 1. The score for adhesions of anastomotic sites in rabbits which underwent tracheal resection and anastomosis with/without additional treatment. G1: Control, tracheal resection and anastomosis alone; G2: tracheal resection and anastomosis plus application of fibrin glue; G3: tracheal resection and anastomosis plus application of platelet-rich plasma jelly. *Significantly different at $p<0.05$ from $G 1$.

results indicate that PRP jelly can significantly reduce postoperative adhesion formation after tracheal reconstruction. Moreover, the amount of exudate at the anastomotic site was significantly lower in the group treated with PRP jelly at 14 days after surgery.

Tracheal narrowing was more prominent in the control and fibrin glue-treated groups compared to that treated with PRP jelly (Table I). PRP jelly application to the anastomotic site resulted in less fibrous stenosis. Stenosis rates between $7 \%$ and $12 \%$ after tracheal reconstruction caused no clinical signs or symptoms in this study. An anastomotic complication increases the risk of postoperative mortality and long-term morbidity. Excessive tension of an anastomotic site causes postoperative complications including granulation, stenosis, and separation $(7,10)$. In this study, the degree of postoperative stenosis after surgery, which would describe the extent of stenosis within individual animals, ranged from $7 \%$ to $44 \%$ (Table I).

On radiographs, normal tracheal walls appeared as thin parallel soft-tissue densities. At the anastomotic site, the tracheal walls appeared slightly thickened, but there were no significant findings. On gross examination, the anastomoses were secure and airtight in all groups. No gaps were observed between tracheal segments. In all cases, mild tracheal stenosis was observed, and tissue repair at the anastomotic site was normal. We observed that fibrin glue and PRP jelly treatment reduced the amount of exudate produced in the early stages of healing. 
A

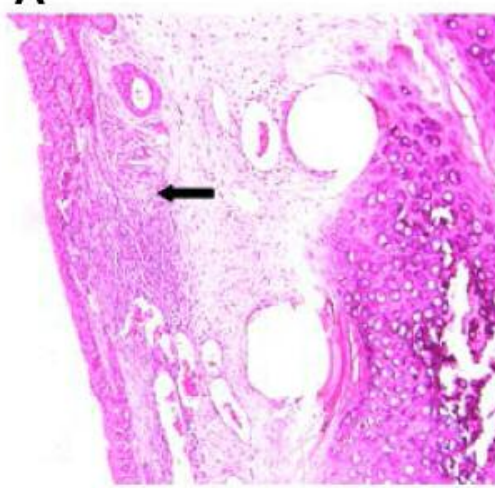

B

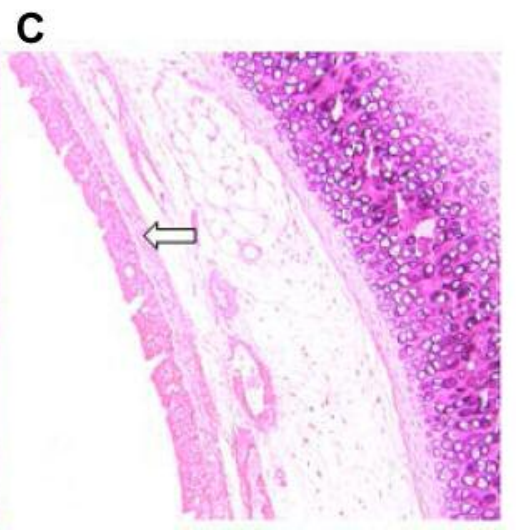

Figure 2. Histopathological view of an anastomosis site at the completion of the study (day 28). A: Marked fibroblastic proliferation, mononuclear cell infiltration, high vessel density and epithelial thickness were observed in the control group (black arrow). B: In the fibrin gIue-treated group, fibroproliferative tissue with fibrosis below the epithelium was less evident. Histopathological examination revealed non-epithelialized epithelium (white arrow). C: The group treated with platelet-rich plasma jelly had completed re-epithelialization in the subepithelial area, with no inflammation (white arrow). Hematoxylin and eosin, $\times 10$.

Table I. Changes at anastomotic site after tracheal resection and anastomosis in rabbits.

\begin{tabular}{|c|c|c|c|c|c|c|}
\hline \multirow{3}{*}{ Group } & \multicolumn{4}{|c|}{ Inner diameter (mm) } & \multicolumn{2}{|c|}{ Narrowing (\%) } \\
\hline & \multicolumn{2}{|c|}{14 Days } & \multicolumn{2}{|c|}{28 Days } & \multirow[t]{2}{*}{14 Days } & \multirow[t]{2}{*}{28 Days } \\
\hline & Normal site & Anastomotic site & Normal site & Anastomotic site & & \\
\hline G1 & $4.1 \pm 0.18$ & $2.52 \pm 0.08^{\mathrm{a}}$ & $5.1 \pm 0.02$ & $3.1 \pm 0.09^{\mathrm{a}}$ & $38.5 \pm 2.8$ & $39.2 \pm 4.7^{a}$ \\
\hline G2 & $4.1 \pm 0.21$ & $3.0 \pm 0.45$ & $5.2 \pm 0.01$ & $4.5 \pm 0.08$ & $27.2 \pm 8.3$ & $13.4 \pm 1.7^{b}$ \\
\hline G3 & $4.1 \pm 0.15$ & $3.6 \pm 0.07^{b c}$ & $5.1 \pm 0.02$ & $4.7 \pm 0.05^{\mathrm{b}}$ & $12.1 \pm 0.7^{b c}$ & $7.8 \pm 0.7^{b c}$ \\
\hline
\end{tabular}

Data are expressed as the mean $\pm \mathrm{SD}(\mathrm{n}=4)$. Significantly different at $p<0.05$ from ${ }^{\mathrm{a}}$ normal site, ${ }^{\mathrm{b}}$ group 1 , ${ }^{\mathrm{c}}$ group 2 . G1: Control, tracheal resection and anastomosis alone; G2: tracheal resection and anastomosis plus application of fibrin glue; G3: tracheal resection and anastomosis plus application of platelet-rich plasma jelly.

Histologically, marked fibroblastic proliferation, mononuclear cell infiltration, high vessel density and epithelial thickness were observed in the control group. In the group treated with fibrin glue, fibroproliferative tissue with fibrosis below the epithelium was less evident. Histopathological examination revealed nonepithelialized epithelium. The PRP jelly-treated group had completed re-epithelialization in the subepithelial area, with no inflammation (Figure 2).

\section{Discussion}

The present study demonstrated the effectiveness of PRP jelly in tracheal resection and anastomosis through histomorphometric evaluations. Anastomotic complications after tracheal resection and reconstruction are common and lead to severe morbidity and mortality $(11,12)$. These complications include granulation at the anastomotic line, stenosis, and separation. In particular, stenosis of the trachea after tracheal reconstruction is primarily caused by excessive tension on the anastomosis $(5,7)$.

PRP jelly application to the anastomotic site resulted in less fibrous stenosis. Stenosis rates between $0 \%$ and $30 \%$ after tracheal reconstruction caused no clinical signs or symptoms in this study. Adhesion scores were lower in the PRP jelly- and fibrin glue-treated groups than in the control group. In addition, the adhesion formation rate was significantly lower in the PRP group compared to the other groups. These results indicate that PRP can significantly reduce postoperative adhesion formation after tracheal reconstruction. Moreover, the amount of exudate at the anastomotic site was significantly reduced in the PRP group at 2 weeks after surgery.

The wound-healing action occurs through a series of phenomena including blood clotting, inflammation, and tissue 
proliferation and maturation (10). Wound healing is regulated by various factors including chemokines, cytokine and growth factors. During wound healing, platelets and macrophages secrete growth factor, initiating the inflammatory process necessary for healing (13). PRP jelly contains proteins and cytokines essential for the wound-healing process (14). Platelets also produce important growth factors such as fibrin, fibronectin, and vitronectin that are responsible for increasing cell mitosis and increasing collagen production (15).

Our results showed that PRP jelly treatment enhanced fibroblast proliferation and collagen production in tracheal healing, unlike the results shown by the other treatment groups. Furthermore, PRP jelly application had effects similar to those of fibrin glue application, better in some respects. Based on our study, autologous PRP jelly contributed to better healing of trachea reconstruction. This improvement in wound healing offers a safe, feasible, and reliable potential new therapeutic option.

\section{Acknowledgements}

This research was supported by Basic Science Research Program through the National Research Foundation of Korea (NRF) funded by the Ministry of Education (2017R1A6A3A11035222)

\section{References}

1 Karliczek A, Benaron DA, Zeebregts CJ, Wiggers T and van Dam GM: Intraoperative ischemia of the distal end of colon anastomoses as detected with visible light spectroscopy causes reduction of anastomotic strength. J Surg Res 152: 288-295, 2009.

2 Tsutsui H1 and Ikeda N: Tracheal resection for the treatment of thyroid cancer invading the trachea-ircumferential sleeve resection followed by end-to-end anastomosis. Nihon Geka Gakkai Zasshi 113: 469-471, 2012.

3 Watanabe S, Takagi K, Nakamura Y and Sakata R: Tracheal release and thymus wrapping of the tracheoplasty anastomosis through mini-sternotomy. Eur J Cardiothorac Surg 25: 287-289, 2004.
4 Ozaki O, Sugino K, Mimura T and Ito K. Surgery for patients with thyroid carcinoma invading the trachea: circumferential sleeve resection followed by end-to-end anastomosis. Surgery 117: 268-271, 1995.

5 Gallagher TQ and Hartnick CJ: Tracheal resection and reanastomosis. Adv Otorhinolaryngol 73: 50-57, 2012.

6 Heldin $\mathrm{CH}$ and Westermark B: Mechanism of action and in vivo role of platelet-derived growth factor. PhysioI Rev 79: 12831316, 1999.

7 Gómez-Caro A, Ausin, P and Boada M: Platelet-rich plasma improves the healing process after airway anastomosis. Interact Cardiovasc Thorac Surg 13: 552-556, 2011.

8 Leary S, Underwood W, Anthony R, Cartner S, Corey D, Grandin T, Greenacre C, Gwaltney-Brant S, McCrackin AM, Meyer R, Miller D, Shearer J and Yanong R: AVMA Guidelines for the Euthanasia of Animals: 2013 edition. Available from https://www.avma.org/KB/Policies/Documents/euthanasia.pdf Last accessed 28/09/2018.

9 Keleidari B, Mahmoudieh M, Bahrami F, Mortazavi P, Aslani RS and Toliyat SA: The effect of vitamin A and vitamin $\mathrm{C}$ on postoperative adhesion formation: A rat model study. J Res Med Sci 19: 28-32, 2014.

10 Werner S and Grose R: Regulation of wound healing by growth factors and cytokines. Physiol Rev 83: 835-870, 2003.

11 Mohsen T, Abou Zeid A, Abdelfattah I, Mosleh M, Adel W and Helal A: Outcome after long-segment tracheal resection: Study of 52 cases. Eur J Cardiothorac Surg 53: 1186-1191, 2018.

12 Auchincloss HG, Wright CD. Complications after tracheal resection and reconstruction: prevention and treatment. J Thorac Dis $8: 160-167,2016$.

13 Schäfer $M$ and Werner S: Transcriptional control of wound repair. Annu Rev Cell Dev Biol 23: 69-92, 2007.

14 Rodriguez IA, Growney, Kalaf EA, Bowlin GL and Swll SA: Platelet-rich plasma in bone regeneration: Engineering the delivery for improved clinical efficacy. Biomed Res Int 2014: 393398, 2014.

15 Marx RE: Platelet-rich plasma. Evidence to support its use. J Oral Maxillofac Surg 62: 489-496, 2004.

Received August 22, 2018

Revised September 28, 2018

Accepted October 2, 2018 